\title{
Modulatory Effect of Cattle on Risk for Lyme Disease
}

\author{
Dania Richter* and Franz-Rainer Matuschka*
}

To determine the effect of cattle on the risk for Lyme disease, we compared the prevalence of spirochete infection in questing vector ticks collected from a pasture with low-intensity cattle grazing with the prevalence in those collected from a site on which no cattle grazed. The presence of cattle limited the prevalence of Borrelia burgdorferi s.I., but not $B$. miyamotoi, in vector ticks. The reintroduction of traditional, nonintensive agriculture in central Europe may help reduce risk for Lyme disease.

$\mathrm{T}$ he tick Ixodes ricinus, which transmits the spirochete agent of Lyme disease, is abundant in the ecotone at a forest's edge. The brushy vegetation there provides a suitable microclimate for these vector ticks as well as food and cover for the animals that serve as hosts for the ticks' subadult stages. Of those, rodents and particular passerine birds serve as reservoirs for the agent of Lyme disease (1-8). Other animals, such as domestic ungulates, serve as hosts for the tick but fail to support the pathogen. These animals exert a zooprophylactic effect on the transmission cycle by diverting ticks from feeding on reservoir-competent hosts. Any tick feeding on these hosts fails to acquire spirochetes and, thereby, fails to contribute to transmission $(9,10)$. In addition, infected ticks may lose the pathogen when feeding on such incompetent hosts (11-14). The ecotone constitutes the focus of transmission, and persons may acquire numerous vector ticks when passing through such a site. The local composition of vertebrate animals serving as hosts to vector ticks determines the risk for infection at that site.

Recently, the European Union and the United Nations Educational, Scientific and Cultural Organization (UNESCO) instituted programs to limit the growth of brush and subsequent reforestation of uncultivated land. These programs specifically target the ecotonal habitat that developed ubiquitously in Europe when cultivating marginal land was considered too labor-intensive and costly.

*Charité Universitätsmedizin Berlin, Berlin, Germany
Toward this end, traditional farming methods are being reimplemented. Domestic ungulates, for example, are being introduced into the ecotone for landscape management. By grazing or browsing on scrub brush and saplings, these animals help maintain open landscapes and simultaneously enhance biodiversity. Although domestic animals may alter the contours of the ecotone, the effect of their presence on the risk for human infection by the agent of Lyme disease has not been defined.

To determine whether the presence of domestic ungulates may reduce risk for human Lyme disease, we compared the prevalence of spirochetal infection in ticks taken from an ecotonal cattle pasture to that in a site in which no cattle were permitted to graze. In addition, we determined the prevalence of the different Lyme disease genospecies in these ticks and that of a distantly related spirochete.

\section{Materials and Methods}

The study site, $\approx 2 \mathrm{~km}^{2}$, is located near the town of Lembach, in the northern Vosges region of France, at the floodplain of the Sauer River. It is part of the Pfälzer Wald-Vosges du Nord biosphere reserve of UNESCO. Scottish Highland cattle were introduced there in 1999 for low-intensity grazing. About 250 cattle are allowed to graze in the floodplain year-round and are rotated on grazing patches enclosed by electric fencing on a total area of $\approx 200 \mathrm{~km}^{2}$. The fence does not constitute a barrier to other vertebrates, such as hedgehogs, foxes, or deer.

On the cattle pasture and at a site $10 \mathrm{~m}$ outside the pasture, questing ticks were sampled by passing a flannel flag over the vegetation; ticks were preserved in $80 \%$ ethanol. We sampled the sites twice, in early May and early June 2005. Ticks were collected from 3 distinct areas within and 2 areas outside the pasture site. Both sites are separated by a path $\approx 4 \mathrm{~m}$ wide. Microscopy was used to identify stage and species of all ticks.

To detect and identify the various spirochetes that may be present in these ticks, the opisthosoma of each was opened, the contained mass of soft tissue was dissected out 
and placed in physiologic saline, and DNA was extracted as described previously (15). Borrelia genospecies were detected and characterized by amplifying and sequencing a 600 -nt fragment of the gene encoding the 16S rRNA by nested PCR (15). Each resulting sequence was compared with sequences of the homologous gene fragment representing each of the validated spirochete genospecies. The following sequences served for comparison: GenBank accession nos. X85196 and X85203 for B. burgdorferi s.s.; X85190, X85192, and X85194 for B. afzelii; X85193, X85199, and M64311 for B. garinii; X98228 and X98229 for B. lusitaniae; X98232 and X98233 for B. valaisiana; AY147008 for B. spielmanii; and AY253149 for B. miyamotoi. A complete match, permitting no more than 2 nt changes, was required.

\section{Results}

To determine the effect of the presence of cattle on the prevalence of spirochetal infection in vector ticks, we sampled questing ticks within a fenced site where cattle grazed, examined them individually for spirochetal DNA by PCR and compared the resulting prevalence to that in ticks sampled from at least $10 \mathrm{~m}$ beyond the fence. Spirochetes infected $\approx 4 \times$ as many nymphs and $\approx 6 \times$ as many adults outside the enclosure as within (Table) (Fisher exact test, $\mathrm{p}<0.0001$ for either developmental stage). The variance between different samples taken within the pasture site and the variance between samples taken outside the pasture were considerably smaller than the variance between the pasture and the nonpasture site. Fewer questing ticks harbored spirochetes where cattle were present than where they were absent.

We then determined whether the presence of cattle affects a spirochetal genospecies more than others. Amplified spirochetal DNA derived from each infected tick was sequenced and the genospecies determined by comparison with published sequences. Where cattle were present, virtually no nymphal or adult ticks were infected by $B$. valaisiana (Fisher exact test, $\mathrm{p}=0.006, \mathrm{p}=0.05$, respectively; Table). The prevalence of $B$. lusitaniae in ticks sampled on the cattle pasture was about one tenth the prevalence in ticks sampled outside the pasture (Fisher exact test, $\mathrm{p}=0.025$ in nymphs, $\mathrm{p}=0.0025$ in adults). $B$. garinii infected about one twentieth as many nymphal ticks and about one eighth as many adult ticks where cattle were present compared with the nonpasture site (Fisher exact test, $\mathrm{p}<0.0001$ for either developmental stage). Whereas the prevalence of $B$. afzelii in nymphal ticks appeared to be not significantly affected by the presence of cattle, its prevalence in adult ticks was reduced to approximately one quarter (Fisher exact test, $p=0.0019$ ). Too few ticks infected by B. burgdorferi s.s. or B. spielmanii were sampled to permit comparison. In contrast to the prevalence of Lyme disease spirochetes, the prevalence of $B$. miyamotoi, an ixodid-borne member of relapsing fever spirochetes, was similar, regardless of the presence of cattle. We conclude that the presence of cattle appears to reduce the prevalence of Lyme disease spirochetes in vector ticks but appears not to influence that of a distantly related spirochete.

\section{Discussion}

A vertebrate animal originally was said to be zooprophylactic if its presence in an endemic site serves to divert invertebrate vectors of a human pathogen from feeding on people (16). The concept was extended for Lyme disease to include more specific diversionary effects on the force of transmission of the pathogen, including diversion of the vector tick from feeding on potential reservoir hosts, transportation of vector ticks away from the venue of transmission, or elimination of infection from a previously infected tick (11). Lizards, for example, fail to support Lyme disease spirochetes, and the prevalence of infection in questing vector ticks appears reduced where such hosts are abundant $(2,9)$. Ticks may detach from birds in nonsuitable habitats. Zooprophylaxis may affect the prevalence of infection in vector ticks by various factors.

Wild ungulates are generally thought to contribute to risk for Lyme disease, mainly by supporting the population of vector ticks. Indeed, adult I. dammini (or scapularis) ticks in the northeastern United States appear to preferably feed on white-tailed deer (17). Because they serve as definitive host for the tick, density of deer correlates with tick abundance $(18,19)$. Reduction or elimination of deer

\begin{tabular}{|c|c|c|c|c|c|c|c|c|c|c|c|}
\hline \multirow[b]{2}{*}{$\begin{array}{l}\text { Cattle } \\
\text { in site }\end{array}$} & \multicolumn{4}{|c|}{ Ticks examined } & \multicolumn{7}{|c|}{$\%$ Ticks infected by Borrelia } \\
\hline & Stage & No. & $\begin{array}{c}\% \\
\text { Infected }\end{array}$ & $\pm S D$ & B. afzeli & B. garinii & B. valaisiana & B. burgdorferis.s. & B. lusitaniae & B. spielmanii & B. miyamotoi \\
\hline \multirow[t]{2}{*}{ Present } & Nymph & 238 & 5.5 & 5.8 & 2.9 & 0.4 & 0 & 0.4 & 0.4 & 0 & 1.3 \\
\hline & Adult & 136 & 5.9 & 3.7 & $3.7 \dagger$ & 1.5 & 0 & 0 & 0.7 & 0 & 0.7 \\
\hline \multirow[t]{2}{*}{ Absent } & Nymph & 95 & 22.1 & 5.8 & 5.3 & 9.5 & $4.2 \dagger$ & 0 & 4.2 & 0 & 0 \\
\hline & Adult & 236 & 40.3 & 2.3 & $13.6 \dagger$ & 12.7 & $3.0 \ddagger$ & 0.4 & 7.6 & 1.3 & 3.0 \\
\hline
\end{tabular}

*SD, standard deviation.

†Includes a tick coinfected with B. garinii.

flncludes 2 ticks coinfected with $B$. garinii. 
on islands or their exclusion by electric fences has resulted in fewer subadult ticks questing in subsequent years; the density of adult ticks, however, has declined only slowly (20-23). In contrast, a simulation model indicates that the density of ticks may be more sensitive to the availability of hosts for the subadult stages than to that of hosts for adult ticks (24). In Europe, diverse medium-sized and large mammals, such as hares, hedgehogs, and foxes, constitute alternative hosts for adult ticks (25-27). As a result, any association between the abundance of vector ticks and the density of deer may be less profound in Europe than in eastern North America.

Domestic as well as wild ungulates exert a powerful zooprophylactic effect on Lyme disease spirochetes because they are incompetent for these pathogens. Not only do noninfected ticks that feed on these mammals fail to acquire spirochetes, but also infected ticks lose their infection during the course of such a bloodmeal $(10,12,28,29)$. The sensitivity to the bacteriolytic activity of the complement pathway may determine the incompetence of a host (30). Cattle support the feeding of numerous ticks of all stages (10) and thereby divert subadult vector ticks from feeding on reservoir-competent hosts. A simulation model indicated that the availability of incompetent hosts for subadult tick stages would reduce prevalence of infection (24). Our observation that questing nymphal and adult ticks collected on a cattle pasture were less likely to be infected by Lyme disease spirochetes than were vector ticks collected outside the pasture supports this model. In addition, cattle and other domestic ungulates may modify the ecotonal vegetation by their grazing. This would render the microclimate more arid and, thereby, less suitable for the survival of ticks. Indeed, during equal time periods, we collected approximately half as many questing ticks where cattle were present as we did where cattle were absent (1 tick every 4 minutes vs. 2 minutes, respectively). Grazing cattle may also deplete reservoir-competent rodents and birds foraging in this ecotone of their food source and of cover from predators. By reducing the availability of potential reservoir hosts, additional subadult stages are diverted to feed on incompetent domestic ungulates. Whether the effect of cattle on the prevalence of spirochete infection in questing vector ticks results from the inability of cattle to support the pathogen or from their effect on tick habitat and reservoir rodents remains to be determined. In addition to their parasitologic effects, certain zooprophylactic animals may modify the ecotonal landscape in ways that affect transmission of the agent of Lyme disease.

Although the presence of cattle appears to suppress the prevalence of the various genospecies of Lyme disease spirochetes, that of B. miyamotoi is not affected. It is more closely related to spirochetes that cause relapsing fever than to spirochetes that cause Lyme disease (31). No disease relationship has yet been demonstrated for this microbe. B. miyamotoi spirochetes infect $\approx 2 \%-5 \%$ of questing nymphal or adult $I$. ricinus-like ticks, regardless of location $(15,32)$. The prevalence of Lyme disease spirochetes, on the other hand, varies from zero to $\approx 50 \%$, depending on the local composition of reservoir populations. Our observation that the prevalence of B. miyamotoi is insensitive to the presence of cattle corroborates reports on transovarial transmission (32) and suggests that this spirochete may cycle solely within the vector population.

The European Union and UNESCO support various policies for the deintensification of agriculture. The European Union's Biodiversity Action Plan for Agriculture, for example, recognizes that traditional nonintensive agriculture, as it was practiced for centuries until only a few decades ago, maintained an enormous species variety in wild and domestic flora and fauna (33). By encouraging extensive agricultural practices, such programs aim to prevent further abandonment of marginal farmland and the subsequent development of scrub brush and forests that support relatively few species. Management of previous fallow land as low-intensity pastures for robust varieties of cattle, sheep, goats, horses, or pigs helps maintain or reestablish not only open landscapes but also high nature value habitats (high biodiversity achieved by extensive farming). Our finding that the prevalence of Lyme disease spirochetes in questing vector ticks is significantly reduced on such pastures suggests that enhanced biodiversity may also contribute directly to human health. Conclusions derived from a mathematical model similarly suggest that a species-rich composition of vertebrates that serve as hosts for vector ticks would decrease the prevalence of spirochete-infected ticks by diluting the effect of reservoir-competent hosts (34). Efforts aimed at extensive landscape management may help to reduce risk for Lyme disease in central Europe.

We conclude that cattle appear to modulate risk for Lyme disease by reducing the prevalence of Lyme disease spirochetes in vector ticks; they do not, however, influence prevalence of a distantly related spirochete. Our observations were conducted in a high nature-value habitat that included an extensively managed cattle pasture. In addition to the direct parasitologic effects, certain zooprophylactic animals may modify the landscape in ways that affect the transmission of Lyme disease spirochetes. Removal of ground-covering vegetation may reduce the force of transmission of this pathogen by reducing local rodent density, thereby diverting vector ticks to reservoirincompetent cattle. Zooprophylaxis may include environmental modification. Our finding that the prevalence of B. miyamotoi is insensitive to the presence of cattle suggests that this spirochete may be perpetuated differently 
than are Lyme disease spirochetes. Extensive landscape management in central Europe may help reduce risk for Lyme disease.

\section{Acknowledgments}

We thank Daniela Schlee, Mandy Pötter, and Udo Bischoff for excellent technical assistance.

This study was supported by grant Ma 942/10-2 from the Deutsche Forschungsgemeinschaft.

Dr Richter is an associate research scientist at the medical school Charité Universitätsmedizin Berlin. Her research interests focus on the immunologic and molecular interface of the hostvector-pathogen relationship in the epizootiology of tickborne diseases.

Dr Matuschka is a university professor at the medical school Charité Universitätsmedizin Berlin and head of the parasitology laboratory. His research focuses on transmission cycles of zoonotic diseases.

\section{References}

1. Donahue JG, Piesman J, Spielman A. Reservoir competence of whitefooted mice for Lyme disease spirochetes. Am J Trop Med Hyg. 1987;36:92-6.

2. Matuschka F-R, Fischer P, Heiler M, Richter D, Spielman A. Capacity of European animals as reservoir hosts for the Lyme disease spirochete. J Infect Dis. 1992;165:479-83.

3. Matuschka F-R, Endepols S, Richter D, Spielman A. Competence of urban rats as reservoir hosts for Lyme disease spirochetes. J Med Entomol. 1997;34:489-93.

4. Matuschka F-R, Allgöwer R, Spielman A, Richter D. Characteristics of garden dormice that contribute to their capacity as reservoirs for Lyme disease spirochetes. Appl Environ Microbiol. 1999;65:707-11.

5. Olsen B, Jaenson TG, Bergstrom S. Prevalence of Borrelia burgdorferi sensu lato-infected ticks on migrating birds. Appl Environ Microbiol. 1995;61:3082-7.

6. Richter D, Spielman A, Komar N, Matuschka F-R. Competence of American robins as reservoir hosts for Lyme disease spirochetes. Emerg Infect Dis. 2000;6:133-8.

7. Tälleklint L, Jaenson TG. Transmission of Borrelia burgdorferi s.l. from mammal reservoirs to the primary vector of Lyme borreliosis, Ixodes ricinus (Acari: Ixodidae), in Sweden. J Med Entomol. 1994;31:880-6.

8. Ginsberg HS, Buckley PA, Balmforth MG, Zhioua E, Mitra S, Buckley FG. Reservoir competence of native North American birds for the Lyme disease spirochete, Borrelia burgdorferi. J Med Entomol. 2005;42:445-9.

9. Lane RS, Loye JE. Lyme disease in California: Interrelationship of Ixodes pacificus (Acari: Ixodidae), the western fence lizard (Sceloporus occidentalis), and Borrelia burgdorferi. J Med Entomol. 1989;26:272-8.

10. Matuschka F-R, Eiffert H, Ohlenbusch A, Richter D, Schein E, Spielman A. Transmission of the agent of Lyme disease on a subtropical island. Trop Med Parasitol. 1994;45:39-44.

11. Matuschka F-R, Spielman A. Loss of Lyme disease spirochetes from Ixodes ricinus ticks feeding on European blackbirds. Exp Parasitol. 1992;74:151-8.
12. Matuschka F-R, Heiler M, Eiffert H, Fischer P, Lotter H, Spielman A. Diversionary role of hoofed game in the transmission of Lyme disease spirochetes. Am J Trop Med Hyg. 1993;48:693-9.

13. Telford SR, Mather TN, Moore SI, Wilson ML, Spielman A. Incompetence of deer as reservoirs of the Lyme disease spirochete. Am J Trop Med Hyg. 1988;39:105-9.

14. Jaenson TG, Tälleklint L. Incompetence of roe deer as reservoirs of the Lyme borreliosis spirochete. J Med Entomol. 1992;29:813-7.

15. Richter D, Schlee DB, Matuschka F-R. Relapsing fever-like spirochetes infecting European vector tick of Lyme disease agent. Emerg Infect Dis. 2003;9:697-701.

16. Russell PF, West LS, Manwell RD, MacDonald G. Practical malariology, 2nd ed. London: Oxford University Press; 1963.

17. Piesman J, Spielman A, Etkind P, Ruebush TK, Juranek DD. Role of deer in the epizootiology of Babesia microti in Massachusetts, USA. J Med Entomol. 1979;15:537-40.

18. Rand PW, Lubelczyk C, Lavigne GR, Elias S, Holman MS, Lacombe $\mathrm{EH}$, et al. Deer density and the abundance of Ixodes scapularis (Acari: Ixodidae). J Med Entomol. 2003;40:179-84.

19. Wilson ML, Adler GH, Spielman A. Correlation between abundance of deer and that of the deer tick, Ixodes dammini (Acari: Ixodidae). Ann Entomol Soc Am. 1985;78:172-6.

20. Daniels TJ, Fish D. Effect of deer exclusion on the abundance of immature Ixodes scapularis (Acari: Ixodidae) parasitizing small and medium-sized mammals. J Med Entomol. 1995;32:5-11.

21. Rand PW, Lubelczyk C, Holman MS, Lacombe EH, Smith RP. Abundance of Ixodes scapularis (Acari: Ixodidae) after the complete removal of deer from an isolated offshore island, endemic for Lyme disease. J Med Entomol. 2004;41:779-84.

22. Stafford KC. Reduced abundance of Ixodes scapularis (Acari: Ixodidae) with exclusion of deer by electric fencing. J Med Entomol. 1993;30:986-96.

23. Wilson ML, Telford SR, Piesman J, Spielman A. Reduced abundance of immature Ixodes dammini (Acari: Ixodidae) following elimination of deer. J Med Entomol. 1988;25:224-8.

24. Van Buskirk J, Ostfeld RS. Controlling Lyme disease by modifying the density and species composition of tick hosts. Ecol Appl. 1995;5:1133-40.

25. Sréter T, Széll Z, Varga I. Ectoparasite infestations of red foxes (Vulpes vulpes) in Hungary. Vet Parasitol. 2003;115:349-54.

26. Tälleklint L, Jaenson TGT. Infestation of mammals by Ixodes ricinus ticks (Acari: Ixodidae) in south-central Sweden. Exp Appl Acarol. 1997;21:755-71.

27. Walter G, Liebisch A. Untersuchungen zur Biologie und Verbreitung von Zecken (Ixodoidea, Ixodidae) in Norddeutschland. III. Ixodes ricinus (Linnaeus 1758). Z Angew Zool. 1980;67:449-76.

28. Jaenson TGT, Tälleklint L. Incompetence of roe deer as reservoirs of the Lyme borreliosis spirochete. J Med Entomol. 1992;29:813-7.

29. Telford SR, Mather TN, Moore SI, Wilson ML, Spielman A. Incompetence of deer as reservoirs of the Lyme disease spirochete. Am J Trop Med Hyg. 1988;39:105-9.

30. Kurtenbach K, De Michelis S, Etti S, Schäfer SM, Sewell H-S, Brade $\mathrm{V}$, et al. Host association of Borrelia burgdorferi sensu lato-the key role of host complement. Trends Microbiol. 2002;10:74-9.

31. Fukunaga M, Koreki Y. The flagellin gene of Borrelia miyamotoi sp. nov. and its phylogenetic relationship among Borrelia species. FEMS Microbiol Lett. 1995;134:255-8.

32. Scoles GA, Papero M, Beati L, Fish D. A relapsing fever group spirochete transmitted by Ixodes scapularis ticks. Vector Borne Zoonotic Dis. 2001;1:21-34.

33. Biodiversity action plan for agriculture. Communication from the commission to the council and the European parliament, vol. III; 2001 [cited 2006 Oct]. Commission of the European communities, Brussels, Belgium [online]. Available from http://ec.europa.eu/agriculture/envir/biodiv/162_en.pdf 
34. LoGiudice K, Ostfeld RS, Schmidt KA, Keesing F. The ecology of infectious disease: Effects of host diversity and community composition on Lyme disease risk. Proc Natl Acad Sci U S A. 2003;100:567-71.
Address for correspondence: Dania Richter, Abteilung Parasitologie, Institut für Pathologie, Charité Universitätsmedizin Berlin, Malteserstraße 74-100, 12249 Berlin, Germany; email: drichter@charite.de
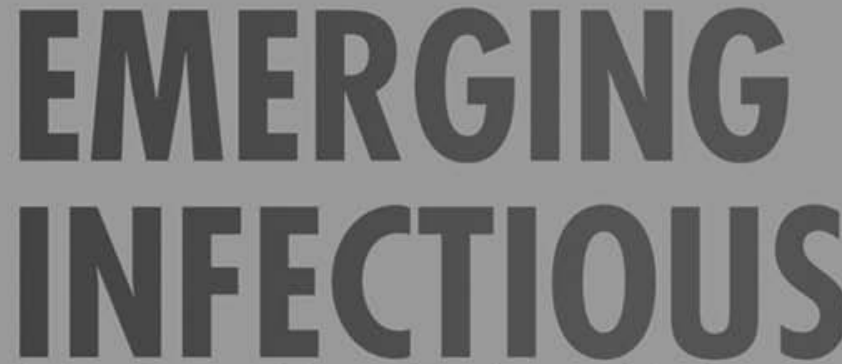

A Peer-Reviewed Journal Tracking and Analyzing Disease Trends
EID

Online

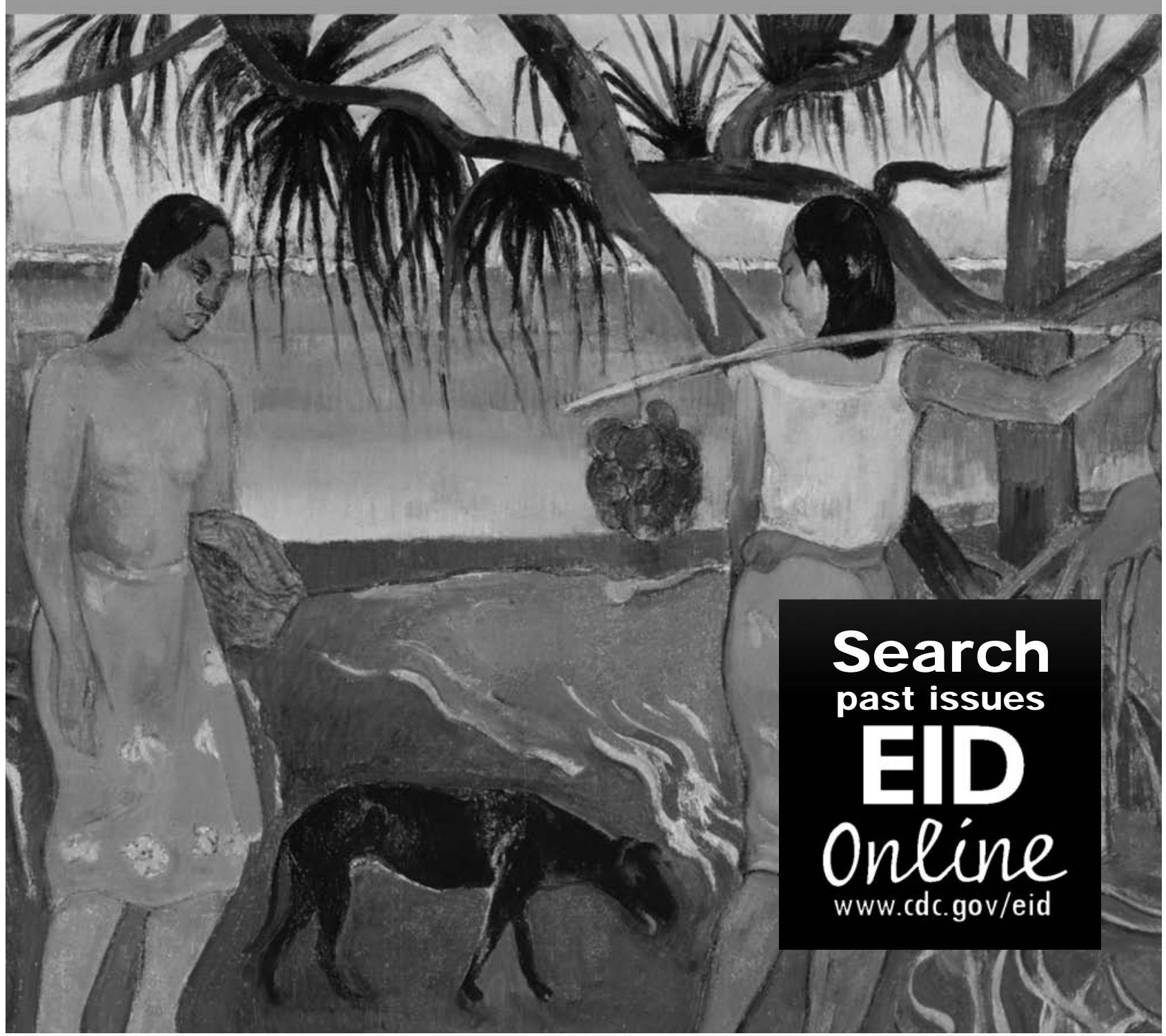

